\title{
Clinica Dermosifilopatica della R. Universita di Roma.
}

Oktober 1913.

Campana, R. Tuberkulose der Luftwege: verschiedene Formen; Vergleichungdesanatomischen Zustandes; Diagnose; Hämoptoebei Tuberkulösen. p. $91 \mathrm{ff}$.

Ausführliche mit zahlreichen Illustrationen versehene Arbeit, nicht zum kurzen Referat geeignet.

Galimberti, A. Hemiatrophia facialis und Hemiatrophia dorsalis der gegenüberliegenden Seite. p. 117.

Kurze kasuistische Mitteilung des in der Überschrift angeführten Falles.

Lollini, Livia. Lepra. p. 121.

Mitteilung eines Falles von Lepra mit Bemerkungen über Untersuchungen Campanas. Fritz Juliusberg (Posen).

\section{Dermatologie.}

Russische Monatescbrift für Haut- und venerische Krankheiten.

Bd. II. H. 8. August 1913.

1. Lebedew, A. I. Über die Wirkung des Salvarsans auf kapillare Gefäße. p. 767.

Der Verfasser studierte die Wirkung der Salvarsaninfusionen auf normale Kaninchen. Toxische Dosen ( 015 pro Kilo) hatte Zerstörung des Endothels der Kapillaren, Hämorrhagien und zahlreiche Thrombosen (rote Thromben) zur Folge. Therapeutische Dosen $(0.01-0.015$ pro Kilo) riefen Hyperämie, Dilatation der Kapillaren, manchmal Thrombose, Blutung in den Nieren, Veränderungen des Endothels hervor. Nach dreimaliger Anwendung der therapeutischen Dosis in wöchentlichen Zwischenräumen entstanden in den Nieren und Lungen fast dieselben Veränderungen wie nach der Dosis toxica. Wahrscheinlich ist diese organotrope Wirkung durch den Arsengehalt des Salvarsans zu erklären. Es ist besser, hohe "sterilisierende" Dosen des Salvarsans zu vermeiden.

2. Bogoljepow, A. A. Zur Frage der Ätiologie der Syphilis. p. 775. (Schluß folgt.)

Auf Grund zahlreicher, genauer Untersuchungen hält Verf. die Anwesenheit mykotischer Formen (mikroskopisch und kulturell) in verschiedenen Stadien der Syphilis für bewiesen und will die Spirochaeta pallida nur als ein Stadium im Entwicklungszyklus dieses Pilzes ansehen. 